\title{
Gestión de la energía dietaría en la reducción de costo de alimentación en la producción de huevos
}

\author{
Diet energy management in reducing feed cost in egg production
}

Gestão da energia da dieta na redução do custo da ração na produção de ovos

\author{
Elías Salvador Tasayco \\ elias.salvador@unica.edu.pe \\ https://orcid.org/0000-0002-4298-7144
}

\author{
Sandra Jackeline Bonifacio Huallanca \\ 20171497@unica.edu.pe \\ https://orcid.org/0000-0002-6264-5440
}

Facultad de Medicina Veterinaria y Zootecnia-Universidad Nacional “San Luis Gonzaga”, Ica, Perú

Artículo recibido 2 de septiembre 2021 / Arbitrado y aceptado 6 de octubre 2021 / Publicado 13 de diciembre 2021

\section{RESUMEN}

Precios altos del maíz y soya incrementa en más de $30 \%$ el costo de la dieta. La energía metabolizable (EM) es el componente clave de dicho costo. Reevaluar el nivel de EM permite definir el óptimo consumo de alimento y energía para mejorar el costo de alimentación. El objetivo fue determinar el efecto de tres niveles de EM sobre la respuesta productiva, calidad de huevo y margen sobre costo de alimentación de gallinas de postura. Se utilizaron 192 gallinas de la línea DEKALB de 80 semanas de edad, bajo un Diseño completo al Azar. Los niveles de EM: 2650, 2750 y $2850 \mathrm{Kcal} / \mathrm{Kg}$, con cuatro repeticiones. Se evaluó la respuesta productiva, calidad de huevo y margen sobre costo de alimentación. Para los análisis estadísticos se usó el procedimiento GLM de SAS v 9.4. La calidad de huevo no fue afectada. El consumo de alimento aumento significativamente $(P<0.05)$ conforme se redujo el nivel de EM en la dieta. La ganancia de peso vivo fue mayor con el nivel más bajo de EM. El margen sobre el costo de alimentación para masa de huevo fue más alto con el nivel de $2650 \mathrm{Kcal}$ de $\mathrm{EM} / \mathrm{Kg}$ con una retribución económica $+6.99 \%$.

Palabras clave: Ponedoras; Energía metabolizable; Margen económico

ABSTRACT

\section{RESUMO}

High prices of corn and soybeans increase the cost of the diet by more than 30\%. Metabolizable energy (ME) is the key component of that cost. Reassessing the level of ME allows to define the optimal consumption of food and energy to improve the cost of feeding. The objective was to determine the effect of three levels of $\mathrm{ME}$ on the productive response, egg quality and margin on feeding cost of laying hens. 192 hens of the 80-week-old DEKALB line were used, under a complete Random Design. ME levels: 2650, 2750 and 2850 $\mathrm{Kcal} / \mathrm{Kg}$, with four repetitions. The productive response, egg quality and margin on feed cost were evaluated. For statistical analyzes, the GLM procedure of SAS v 9.4 was used. Egg quality was not affected. Food consumption increased significantly $(P<0.05)$ as the level of ME in the diet decreased. Live weight gain was greater with the lowest level of ME. The margin on the cost of feeding for egg dough was higher with the level of $2650 \mathrm{Kcal}$ of ME / Kg with an economic retribution $+6.99 \%$.

Key words: Layers; Metabolizable energy; Economic margin
Os altos preços do milho e da soja aumentam o custo da dieta em mais de 30\%. A energia metabolizável (EM) é o principal componente desse custo. Reavaliar o nível de EM permite definir o consumo ideal de alimentos e energia para melhorar o custo da alimentação. O objetivo foi determinar o efeito de três níveis de EM na resposta produtiva, qualidade dos ovos e margem no custo alimentar de poedeiras comerciais. Foram utilizadas 192 galinhas da linhagem DEKALB de 80 semanas, sob um Desenho Aleatório completo. Níveis de EM: 2650, 2750 e 2850 Kcal / $\mathrm{Kg}$, com quatro repetições. A resposta produtiva, a qualidade do ovo e a margem sobre o custo da ração foram avaliadas. Para análises estatísticas, o procedimento GLM do SAS v 9.4 foi usado. A qualidade do ovo não foi afetada. $O$ consumo de alimentos aumentou significativamente ( $P$ $<0,05$ ) conforme o nível de EM na dieta diminuiu. $O$ ganho de peso vivo foi maior com o menor nível de EM. A margem sobre o custo da alimentação da massa de ovo foi maior com o nível de $2650 \mathrm{Kcal}$ de EM / Kg com uma retribuição econômica de $+6,99 \%$.

Palavras-chave: Oedeiras; Energía metabolizável; Margem econômica 


\section{INTRODUCCIÓN}

Decidir el nivel de EM de la dieta, aporte de EM/gallina/día y el consumo de alimento optimo es de gran importancia económica en la industria avícola. No es lo mismo formular una dieta con EM para una característica productiva que para maximizar el margen económico sobre costo de alimentación para masa de huevo. Es un tema complejo y depende de diversos factores, por lo que siempre debe estar en discusión y reevaluación para tomar la mejor decisión a nivel de la avicultura de producción de huevos a nivel comercial, más aún bajo condiciones de aumento del precio del maíz y soya que son las principales fuentes de energía y aminoácidos.

Estos tres criterios de decisión están relacionados. La literatura reporta algunos datos de esta relación. Si bien según Hill et al. (1), inicialmente reporto que las gallinas ajustan su consumo de alimento para satisfacer sus necesidades energéticas $y$, en consecuencia, un aumento en la concentración energética de la dieta debería reducir el consumo de alimento proporcionalmente. Pero, según otros estudios indican algunas diferencias. Cuando se cambia la concentración de energía de la dieta han dado como resultados contrastantes con respecto a la ingesta de energía, el rendimiento productivo y la tasa de conversión alimenticia (CA) de las gallinas (2).

Bajo las condiciones actuales, con el incremento de los precios del maíz y aceite como las principales fuentes de energía surge la necesidad de reevaluar estos conceptos. En ciertos casos con el propósito de reducir costo de la dieta se reduce grandemente o excluye la inclusión de aceites en las dietas, según mi opinión es una decisión errónea. Generalmente al aumentar el contenido de EM de la dieta, se agrega más grasa y la grasa suplementaria podría mejorar la utilización de otros componentes de la dieta $(3,4)$.

Las aves alimentadas con dietas más bajas en energía tienden a tener proporciones de conversión alimenticia (CA) más bajas, ya que se necesita más alimento para proporcionar la energía necesaria para el crecimiento, mantenimiento y / o producción de huevos (5).

Peguri y Coon (6) informaron una disminución del $5 \%$ en el consumo de alimento y producción de huevos similar cuando la AMEn de la dieta se incrementó de 2,700 a $2,910 \mathrm{kcal} / \mathrm{kg}$ (un aumento del $8 \%$ ). En base a estos resultados se podría analizar si esa reducción del consumo justifica la respuesta económica y calcular o hacer una estimación si aumentar el nivel de EM podría convenir en la rentabilidad. Porque, si con el mismo nivel de EM se mantiene la respuesta productiva entonces sería conveniente encontrar el nivel óptimo compatible con la máxima rentabilidad bajo las condiciones de campo. Sin embargo, otros estudios han encontrado mejoras significativas cuando se aumentó el nivel de EM de la dieta.

Las dietas energéticas altamente concentradas, sin embargo, son costosas y podrían reducir la eficiencia alimenticia para la producción de huevos, porque parte de la energía ingerida se dirige a la deposición de grasa y la ganancia de peso corporal (7). 
Muchos estudios han evaluado su efecto sobre la respuesta productiva, pero a la vez no menciona su efecto sobre el margen económico. En esta línea el objetivo del estudio fue determinar el efecto de tres niveles de EM sobre la respuesta productiva, calidad de huevo y margen sobre costo de alimentación para masa de huevo de gallinas de postura.

\section{MATERIALES Y MÉTODOS}

El estudio se realizó en la unidad experimental de nutrición en gallinas de postura del Laboratorio de Nutrición R \& D de la Facultad de Medicina Veterinaria y Zootecnia de la Universidad Nacional "San Luis Gonzaga". El periodo de ejecución fue de junio a agosto del 2021. La temperatura promedio del galpón fue de $17^{\circ} \mathrm{C}$. Se utilizaron 192 gallinas de postura de la línea genética DEKALB BROWN, de 80 semanas de edad, criadas en el sistema de jaulas. Se establecieron tres niveles de EM como tratamientos: 2650, 2750 y 2850 Kcal/ Kg. Se utilizó un Diseño Completo al Azar con
3 tratamientos y 4 repeticiones. Las variables evaluadas fueron: producción de huevo, consumo de alimento, conversión alimenticia, consumo de EM, eficiencia energética, masa de huevo, peso vivo final de las gallinas, ganancia de peso vivo, \% de yema, \% de albumen, índice de yema y \% de cascara del huevo, así como el margen sobre costo de alimentación por kg de masa de huevo y retribución económica. Se realizaron análisis estadístico de varianza, Kruskal-Wallis y Tukey, con el procedimiento GLM de SAS v 9.4 (8). Se estableció un nivel de significancia de 0.05 .

\section{RESULTADOS Y DISCUSIÓN}

En la Tabla 1, se observa que la producción de huevo, índice de conversión alimenticia y consumo de EM no fueron afectados significativamente $(P>0.05)$, mientras que se encontró diferencias estadísticas $(P<0.05)$ en el consumo de alimento. El consumo de alimento aumentó significativamente $(\mathrm{P}<0.05)$ conforme se redujo el nivel de EM en la dieta.

Tabla 1. Efecto de tres niveles de EM en la dieta sobre la producción de huevos (PRH), consumo de alimento (CA), índice de conversión alimenticia (ICA) y consumo de EM (CEM) de gallinas de postura.

\begin{tabular}{lcccc}
\hline $\begin{array}{r}\text { Tratamientos } \\
\text { (EM, Kcal/Kg) }\end{array}$ & $\begin{array}{r}\text { PRH } \\
(\%)\end{array}$ & $\begin{array}{c}\text { CA } \\
\text { (g/día) }\end{array}$ & $\begin{array}{r}\text { ICA } \\
\text { (g/g) }\end{array}$ & $\begin{array}{c}\text { CEM } \\
\text { (Kcal/día) }\end{array}$ \\
\hline 2650 & $91.36 \pm 6.96$ & $135.60 \mathrm{a} \pm 7.24$ & $2.30 \pm 0.11$ & $359.35 \pm 19.20$ \\
2750 & $88.63 \pm 7.70$ & $126.99 \mathrm{ab} \pm 9.19$ & $2.27 \pm 0.10$ & $349.24 \pm 25.29$ \\
2850 & $87.20 \pm 3.13$ & $120.02 \mathrm{~b} \pm 3.86$ & $2.16 \pm 0.07$ & $342.07 \pm 11.00$ \\
Probabilidad & & & & \\
P-value & 0.4638 & 0.0380 & 0.1943 & 0.4785 \\
\hline
\end{tabular}

$\mathrm{P}<0.05=$ diferencia significativa .

$(a, b)=$ Letras como superíndices diferentes entre promedios para cada variable indica diferencia significativa. 
En la Tabla 2, se observa que el índice de eficiencia energética (conversión calórica), masa de huevo y peso vivo final no fue afectado significativamente $(P>0.05)$. La ganancia de peso fue significativamente más alta $(\mathrm{P}<0.05)$ para el grupo de gallinas que consumieron la dieta con el más bajo nivel de EM comparado a los otros dos tratamientos con mayores niveles de EM de más baja ganancia de peso.

Tabla 2. Efecto de tres niveles de EM en la dieta sobre la eficiencia energética (EE), masa de huevo $(\mathrm{MH})$, peso vivo final (PVF) y ganancia de preso (GP) de gallinas de postura.

\begin{tabular}{lcccc}
\hline $\begin{array}{r}\text { Tratamientos } \\
\text { (EM, Kcal/Kg) }\end{array}$ & $\begin{array}{c}\text { EE } \\
\text { (Kcal/Kg) }\end{array}$ & $\begin{array}{c}\text { MH } \\
\text { (g/día) }\end{array}$ & $\begin{array}{c}\text { PVF } \\
\text { (g/ave) }\end{array}$ & $\begin{array}{c}\text { GP } \\
\text { (g/ave/día) }\end{array}$ \\
\hline 2650 & $6.10 \mathrm{a} \pm 0.29$ & $59.09 \mathrm{a} \pm 5.44$ & $2065 \mathrm{a} \pm 58.13$ & $4.64 \mathrm{a} \pm 1.25$ \\
2750 & $6.24 \mathrm{a} \pm 0.30$ & $56.06 \mathrm{a} \pm 5.34$ & $2013 \mathrm{a} \pm 54.23$ & $1.81 \mathrm{~b} \pm 0.87$ \\
2850 & $6.17 \mathrm{a} \pm 0.22$ & $55.46 \mathrm{a} \pm 2.58$ & $2039 \mathrm{a} \pm 53.20$ & $2.09 \mathrm{~b} \pm 1.48$ \\
Probabilidad & & & & \\
P-value & 0.7709 & 0.5219 & 0.4484 & 0.0186 \\
\hline
\end{tabular}

$\mathrm{P}>0.05=$ diferencia no significativa

En la Tabla 3, se observa que el porcentaje de yema, porcentaje de albumen, índice de yema y porcentaje de cascara del huevo no fueron afectados significativamente $(P>0.05)$.

Tabla 3. Efecto de tres niveles de EM en la dieta sobre porcentaje de yema (PY), porcentaje de albumen (PA), índice de yema (IY) y porcentaje de cascara (PC) del huevo de gallinas de postura.

\begin{tabular}{lrrrr}
\hline $\begin{array}{r}\text { Tratamientos } \\
\text { (EM, Kcal/Kg) }\end{array}$ & $\begin{array}{r}\text { PY } \\
(\%)\end{array}$ & $\begin{array}{c}\text { PA } \\
(\%)\end{array}$ & $\begin{array}{r}\text { IY } \\
\text { (d/a) }\end{array}$ & $\begin{array}{r}\text { PC } \\
\text { (\%) }\end{array}$ \\
\hline 2650 & $28.43 \pm 1.77$ & $60.61 \pm 2.24$ & $0.40 \pm 0.015$ & $10.95 \pm 0.51$ \\
2750 & $26.51 \pm 1.46$ & $61.59 \pm 1.89$ & $0.39 \pm 0.008$ & $11.88 \pm 0.70$ \\
2850 & $27.37 \pm 0.86$ & $61.24 \pm 1.43$ & $0.38 \pm 0.027$ & $11.38 \pm 0.86$ \\
Probabilidad & & & & \\
P-value & 0.2921 & 0.7788 & 0.5509 & 0.2457 \\
\hline
\end{tabular}

$\mathrm{P}>0.05$ = diferencia no significativa 
En la Tabla 4 se muestra que el margen sobre el costo de alimentación fue más alto con el nivel de $2650 \mathrm{Kcal}$ de $\mathrm{EM} / \mathrm{Kg}$ con una retribución económica $+6.99 \%$.

Tabla 4. Margen económico (ME) sobre costo de alimentación para masa de huevo y retribución económica (RE) de gallinas de postura alimentadas con tres niveles de EM.

\begin{tabular}{ccc}
\hline Tratamientos & ME & RE \\
(EM, Kcal/Kg) & $(\mathbf{S})$ & (\%) \\
\hline 2650 & 1.883 & 106.99 \\
2750 & 1.758 & 100.00 \\
2850 & 1.846 & 105.00 \\
\hline
\end{tabular}

\section{Discusión}

La dieta con $2650 \mathrm{Kcal} / \mathrm{Kg}$ de EM logró numéricamente $4.16 \%$ más de producción de huevos que el grupo con $2850 \mathrm{Kcal} / \mathrm{Kg}$ de EM, sin embargo, esta diferencia no fue significativa $(P=0.4638)$. Este resultado concuerda con otros estudios donde no se observaron diferencias en la producción por el cambio de la EM en la dieta. Un aumento en el contenido de EM de la dieta de 2,680 a $2,810 \mathrm{kcal} / \mathrm{kg}$ (un aumento de $4.8 \%$ ) disminuyó el consumo de alimento en $5.0 \%$ pero la producción de huevos y la masa de huevos no se vieron afectadas (9). Otros estudios como el de Joly y Bougon (10) informaron un aumento del $1,3 \%$ en la producción de huevos, un aumento del $4,5 \%$ en la masa del huevo y un aumento del $5,7 \%$ en la ingesta energética a medida que el contenido energético de la dieta aumentó de 2200 a $2700 \mathrm{kcal}$ de AMEn / kg en gallinas ponedoras pardas de 19 a 68 semanas de edad.

El consumo de alimento fue marcadamente mayor para el grupo de gallinas que consumieron la dieta con $2650 \mathrm{Kcal} / \mathrm{Kg}$ de EM, que consumieron $15.58 \mathrm{~g}$ (12.98\%) más de alimento que las gallinas que consumieron la dieta con $2850 \mathrm{Kcal} / \mathrm{Kg}$ de EM. Esta diferencia en el consumo de alimento se podría explicar parcialmente debido al ambiente con una temperatura promedio de $17^{\circ} \mathrm{C}$ e incluso menor en ciertos días, donde las gallinas aumentaron su consumo de alimento para satisfacer su requerimiento de EM diaria para mantener su nivel de producción y contribuir a mantener su temperatura interna corporal en época de frio. Estos consumos de EM fueron altos y similares para los tres tratamientos (342 a $359 \mathrm{Kcal} /$ día).

Según Hill et al. (1), las gallinas ajustan el consumo de alimento para satisfacer sus necesidades energéticas $y$, en consecuencia, un aumento en la concentración energética de la dieta debería reducir el consumo de alimento proporcionalmente. Sin embargo, los cambios en la concentración de energía de la dieta han dado como resultado resultados contrastantes con respecto a la ingesta de energía, el rendimiento productivo y la tasa de conversión alimenticia (FCR) de las gallinas (2).

La conversión alimenticia ( $\mathrm{g}$ de alimento / $\mathrm{g}$ de masa de huevo) fueron similares para los 
tres tratamientos que oscilaron entre 2.16 y 2.30, y aunque hubo una diferencia de $140 \mathrm{~g}$ de alimento no fueron estadísticamente diferente dado su alta variación, pero que en términos generales fueron índices muy pobres que se explicaría parcialmente por la necesidad de un mayor consumo de alimento bajo condiciones de frio.

Los indicadores de eficiencia energética aparente medida como conversión calórica cuyos valores estuvieron entre 6.10 y 6.24 Mcal de EM/Kg de masa de masa de huevo tampoco fueron diferentes estadísticamente, pero fueron valores de pobre eficiencia ya que algo de energía se direcciona para mantener la temperatura interna corporal.

Para el caso de la masa de huevo no hubo diferencias por el nivel de EM en la dieta. Al respecto Scappaticcio et al. (11) consideran que las discrepancias encontradas en la respuesta de la producción de masa de huevo debido a la concentración de energía de la dieta podrían depender de factores como la composición de los ingredientes y el contenido de nutrientes de la dieta control (contenido de energía y nivel de grasas suplementarias), manejo y condiciones ambientales (humedad y temperatura), línea y edad de las gallinas (peso corporal, tasa de huevos y peso de huevo).

El peso vivo final, si bien fueron similares, oscilando entre 2013 a 2065 g/gallina, sin embargo, el tratamiento con 2650 Kcal de EM/ $\mathrm{Kg}$ logro la más alta ganancia de peso diario. Este resultado es de interés económico ya que bajo nuestras condiciones de crianza al final del ciclo de producción de las gallinas, estas son comercializadas y al obtener una mayor ganancia de peso permite un mayor retorno económico por la venta. Un estudio de Murugesan and Persia (12), encontraron que la reducción en la ingesta de energía entre las aves alimentadas con una dieta con 2880 y $2790 \mathrm{Kcal}$ de EM/Kg (90 kcal / kg) no cambió la energía dividida hacia la producción o el mantenimiento, pero redujo $(P=0.03)$ la energía almacenada (almohadilla de grasa) de las gallinas alimentadas con el nivel bajo de EM. Estos resultados sugieren que la energía se usa siguiendo el patrón de producción y mantenimiento antes de los requerimientos de almacenamiento y que la almohadilla de grasa (almacenamiento de energía) puede ser el indicador más sensible del estado energético de la dieta a corto plazo en gallinas ponedoras Hy-Line W36.

Respecto a la calidad de huevo, las características evaluadas fueron similares, lo que indica que las gallinas que consumieron la dieta con bajo nivel de EM aumentaron su consumo de alimento para obtener la EM similar a los otros tratamientos para mantener la producción y calidad de huevo y en este caso lograr una mayor ganancia de peso.

Finalmente, los resultados indican un mayor margen económico sobre costo de alimentación para masa de huevo y mejor retribución económica con el nivel más bajo de $2650 \mathrm{Kcal}$ de EM/Kg. Esta variable es de importancia y se debe analizar este resultado para la toma de decisiones y la posibilidad de considerarla como una alternativa u estrategia en la industria comercial que tengan similitud con las condiciones en que se desarrolló este estudio como línea genética, edad de las aves, temperatura ambiente y tomar en cuenta las características de la dieta balanceada 
utilizada. Aunque continuaran los estudios de esta línea para más información y consolidar los hallazgos, así como ir adaptando en la práctica comercial para su validación.

\section{CONCLUSIONES}

Bajo las condiciones del estudio, se concluye que el nivel con $2650 \mathrm{Kcal} / \mathrm{Kg}$ de EM en la dieta aumentó el consumo de alimento y consecuentemente una mejor ganancia de peso previa a la venta. El nivel con $2650 \mathrm{Kcal} /$ $\mathrm{Kg}$ de EM maximiza el margen económico sobre costo de alimentación y retribución económica, sin afectar negativamente la respuesta productiva y calidad de huevo. Este hallazgo servirá para tomar decisión apropiada a nivel de granja comercial en época de alza de costo de ingredientes en gallinas de postura de la línea DEKALB Brown de 80 semanas de edad en época de invierno $\left(17^{\circ} \mathrm{C}\right)$.

\section{REFERENCIAS BIBLIOGRÁFICAS}

1. Hill FW, Anderson DL, y Dansky LM. Studies of the energy requirements of chickens 3 . The effect of dietary energy level on the rate and gross efficiency of egg production. Poult. Sci. 1956; 35:54-59

2. Harms RH, Russell GB, y Sloan DR. Performance of four strains of commercial layers with major changes in dietary energy. J. Appl. Poult. Res.2000; 9:535-541

3. Mateos GG, y Sell JL. Influence of graded levels of fat on utilization of pure carbohydrate by the laying hen. J. Nutr. 1980; 110:1894-1903.

4. Mateos GG, y Sell JL. Influence of carbohydrate and supplemental fat source on the metabolizable energy of the diet. Poult. Sci. 1980; 59:2129-2135
5. O'Neill HV M, Mathis G, Lumpkins BS y Bedford MR. The effect of reduced calorie diets, with and without fat, and the use of xylanase on performance characteristics of broilers between 0 and 42 days. Poult. Sci. 2012; 91:1356-1360.

6. Peguri A Y Coon C. Effect of temperature and dietary energy on layer performance. Poult. Sci. 1991; 70:126-138.

7. Pérez-Bonilla A, Novoa S, García J, MohitiAsli M, Frikha M ay Mateos GG. Effect of energy concentration of the diet on productive performance and egg quality of brown egglaying hens differing in initial body weight. Poult. Sci. 2012; 91:3156-3166.

8. STATISTICAL ANALYSIS SYSTEM, INSTITUTE. 2021. User's Guide: Statistics. Version 9.4. Edition. SAS Institute Inc., Cary, NC. USA.

9. Grobas S, Mendez J, De Blas C y Mateos GG. Laying hen productivity as affected by energy, supplemental fat, and linoleic acid concentration of the diet. Poult. Sci. 1999; 78:1542-1551.

10. Joly $P, y$ Bougon $M$. Influence $d u$ niveauénergétique sur les performances des pondeuses à oeufsroux et évolution de l'ingéré en fonction de l'âge. Zême Journée de la Recherche Avicole. 1997; 2:115-120.

11. Scappaticcio R, García J, Fondevila G, de Juan AF, Camara L y Mateos GG. Influence of the energy and digestible lysine contents of the diet on performance and egg quality traits of brown-egg laying hens from 19 to 59 weeks of age. Poult. Sci. 2021; 100:101211. https://doi. org/10.1016/j.psj.2021.101211

12. Murugesan GR, y Persia ME. Validation of the effects of small differences in dietary metabolizable energy and feed restriction in first-cycle laying hens. Poul. Sci. 2013; 92: 12381243 